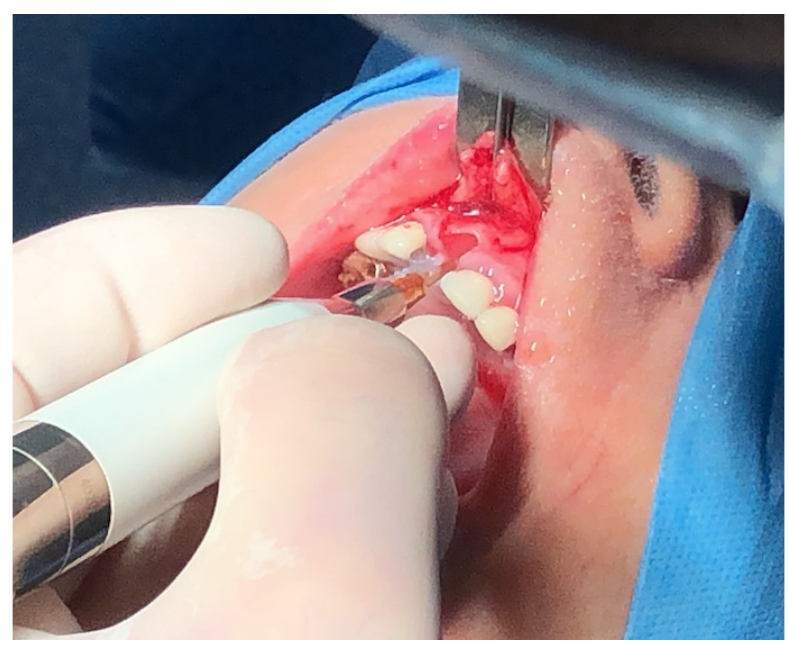

\title{
DENTAL EXTRACTION \& DENOSUMAB. EVALUATION OF PIEZOELECTRIC SURGICAL PROTOCOL FOR MRONJ PREVENTION. PRELIMINARY REPORT.
}

\author{
FRANCESCO DELLA FERRERA ${ }^{1}$, Luca Guaschino, Paolo Appendino ${ }^{1}$, Iorenzo basano ${ }^{1}$, Alessandro Chiarelli ${ }^{1}$ \\ 1 Azienda Ospedaliera Ordine Mauriziano di Torino
}

Funding: The author(s) received no specific funding for this work.

Potential competing interests: The author(s) declared that no potential competing interests exist.

\section{Abstract}

Aim: To evaluate incidence of wound healing complications and MRONJ following tooth extractions in patients treated with Denosumab (Dmab).

Materials and methods: Atraumatic piezosurgery-aided tooth extractions in Dmab treated patients during appropriate drug holiday protocol.

Results: Fortyfive atraumatic tooth extractions in 15 consecutive caucasian patients were performed. High dose regimen patients / Low dose regimen patients : 1/4. Healing of surgical wounds without complications/delay was achieved in 42 extraction sites (93.3\%) in 17 patients (85\%) by 8 weeks evaluation.

Discussion: Despite the little experience existing regarding tooth extraction in exclusive Dmab-treated patients, our preliminary data suggest that atraumatic piezosurgery-aided tooth extractions permit elimination of dental foci without significant complications or delays in Dmab therapy in most cases.

Introduction: Denosumab is a human monoclonal antibody with antiresorptive activity, well-known circulatory half-life and no tissue accumulation. Association with MRONJ (Medication Related Osteonecrosis of the Jaws) is described in literature 
and is similar to bisphosphonates (BP) associated MRONJ. Dmab is currently available with High dose (skeletal related events/SRE in oncology) and Low dose (osteoporosis) regimens ${ }^{1}$. Reports regarding protocols and outcomes of invasive oral procedures (eg. dental extractions) in patients exposed to BP can be found. Same protocols are usually employed in Dmab-treated patients but little experience exist regarding MRONJ prevention efficacy ${ }^{2,3}$. Preliminary data from 20 patients who underwent tooth extraction with a piezoelectric surgical protocol are presented.

Materials and methods: Tooth extraction prognosis data in Dmab-treated patients were collected. Exclusion criteria: history of BP treatment and /or MRONJ, head\&neck radiotherapy. Atraumatic dental extractions with a piezoelectric surgical protocol were performed in local anesthesia and perioperative oral antibiotics. Primary wound closure was achieved by piezoelectric ultrasound alveoloplasty, relieved mucoperiosteal flaps and resorbable sutures. Drug holiday (high dose) and appropriate timing of intervention (low dose) were chosen according to SICMF-SIPMO recommendations ${ }^{2}$. Patients were followed-up for at least 2 months after procedure.

Results: 15 consecutive caucasian patients $\left(20^{\prime \prime}, 139\right.$ mean age 72.8$)$ underwent 45 atraumatic tooth extractions and 1 dental implant removal. Main indications for dental extraction were: advanced periodontitis, periapical abscesses and residual roots. Patients were treated with high dose regimen for SRE (\#4/20\%) and low dose regimen for osteoporosis (\#16/80\%). Main comorbidities were: multiple myeloma, rheumathoid arthritis, diabetes, hypothyroidism. Other identified drugs which could modify MRONJ risk were: methotrexate, steroids (\#2/10\%). Healing of surgical wounds without complications/delay was achieved in 42 extraction sites (93.3\%) in 17 patients (85\%) by 8 weeks evaluation. Three extraction sites in 3 patients needed an extended drug-holiday. Two cases ( 1 high dose, 1 low dose) resolved after medical therapy and observation for postoperative abscess. The last case (high dose) needed a second surgical procedure for bone sequestrum removal 2 months after tooth extraction. All of them reached good healing within two months without recurrence thus far.

Discussion: Despite premedication dental evaluation in Dmab-treated patients, need for dental extractions is quite common in our experience. Several papers state relevance of dental infective-inflammatory diseases rather than their treatment in the aetiology of MRONJ ${ }^{1,2}$. Little experience exist regarding efficacy of specific surgical protocols in managing tooth extraction in Dmab-treated patients despite its potential to replace BP in SRE and osteoporosis management ${ }^{3-4}$. Preliminary data suggest that atraumatic dental extractions with piezoelectric surgical protocol permit elimination of dental foci without significant complications or delays in Dmab therapy in most cases. Healing complications were more frequently associated to high dose regimen patients who are generally considered a population at higher risk of MRONJ development ${ }^{1-3}$.

\section{References:}

1. Owosho A.A. et al. "Osteonecrosis of the jaw in patients treated with denosumab for metastatic tumors to the bone: $A$ series of thirteen patients" J. Cran-Max-Fac Surg 44 (2016) 265e270

2. Di Fede O. et al. "The Dental Management of Patients at Risk of Medication-Related Osteonecrosis of the Jaw: New Paradigm of Primary Prevention" Biomed Res Int. 2018 Sep 16;2018:2684924.

3. Matsumoto A. et al." Primary wound closure after tooth extraction for prevention of medication-related osteonecrosis of the jaw in patients under denosumab" Clin Oral Investig. 2017 Jan;21(1):127-134. 
4. Fusco V, Cabras M, Erovigni F, Dell'Acqua A, Arduino PG, Pentenero M, et al. A multicenter observational study on Medication-Related Osteonecrosis of the Jaw (MRONJ) in advanced cancer and myeloma patients of a cancer network in North-Western Italy. Med Oral Patol Oral Cir Bucal. 2020. doi:10.4317/medoral.24318. 